\title{
An Improved Method for Earthquake Damage Prediction of Highway Subgrade and Pavement Based on Closeness Degree Method
}

\author{
Jia Lee ${ }^{1,2, *}$, Junqi Lin ${ }^{1,2}$, Jinlong Liu ${ }^{1,2}$ \\ ${ }^{1}$ Institute of Engineering Mechanics, China Earthquake Administration, Harbin, China \\ ${ }^{2}$ Key Laboratory of Earthquake Engineering and Engineering Vibration of China Earthquake Administration, Harbin, China \\ Email address: \\ 914517210@qq.com(Jia Lee) \\ ${ }^{*}$ Corresponding author
}

\section{To cite this article:}

Jia Lee, Junqi Lin, Jinlong Liu. An Improved Method for Earthquake Damage Prediction of Highway Subgrade and Pavement Based on Closeness Degree Method. American Journal of Civil Engineering. Vol. 6, No. 4, 2018, pp. 118-122. doi: 10.11648/j.ajce.20180604.12

Received: August 16, 2018; Accepted: September 12, 2018; Published: September 18, 2018

\begin{abstract}
In this article, based on the earthquake damage data of '5.12' Wenchuan Earthquake and the improved method of earthquake damage prediction of highway subgrade and pavement which is obtained by using nonlinear regression method, a new method using closeness degree is given. This new method uses fuzzy recognition to determine the earthquake damage level of certain road section. In order to conduct fuzzy recognition, the membership function of the fuzzy set of the damage grade is also given, after conducting fuzzy recognition for membership function of earthquake damage using closeness degree method, the earthquake damage prediction results of corresponding road sections will be obtained. Finally, an example is given based on the data of earthquake damage in Wenchuan earthquake. The results of the example show that this new method of earthquake damage prediction using closeness degree indeed has higher accuracy, which proves it another effective and practical method for earthquake damage prediction of highway pavement and subgrade. This method also provides a reference for future research on related projects.
\end{abstract}

Keywords: Lifeline Systems, Highway System, Earthquake Damage Estimation, Closeness Degree, Fuzzy Recognition

\section{Introduction}

Highway system is an important part of lifeline system. Once an earthquake occurs, it' s not only related to material transmission and information links, but also related to the timely arrival of disaster relief support, which makes it a veritable life channel indeed. The roads at all levels suffered from the Tangshan earthquake were 228 kilometers long [1]. The California earthquake in 1989 caused serious damage to roads which lead to heavy casualties [2]. The 2008 Wenchuan earthquake caused nearly 28 thousand kilometers of roads to varying degrees of damage [3]. Highway traffic to Wenchuan, Mao county, Beichuan and other disaster-stricken areas was completely interrupted, greatly hindering the advance of rescue teams. New Zealand earthquake in 2011 caused landslides which buried large sections of roads [4], leading to disrupting traffic. In 2013 Lushan earthquake, the S210 line was severely damaged, and the repair time after the earthquake lasted more than 48 hours [5], which increased rescue difficulty and property losses after the earthquake. Obviously, after an earthquake, it is extremely important to be able to clear the main road in time, and this requires to do a good job in advance of the emergency planning- the so-called preparedness against danger. Therefore, a reliable earthquake prediction model can give people a reference when disaster is not yet available. As a part of the basic work of earthquake prevention and disaster reduction for lifeline systems, highway earthquake damage prediction is particularly important.

Based on the earthquake damage prediction method proposed by Japanese scholar Masaru Hoshiya, Chen Yiping [1] carried out earlier research on earthquake damage prediction of highway subgrade and pavement engineering. On the basis of earlier researches, a prediction model of 
earthquake damage is established by using the influence factors of earthquake damage, which is proved to be practical. Jia Xingli [6] put forward the weighting method of highway seismic risk assessment using cloud model AHP, the calculation factors include key factors such as formation lithology and peak acceleration of ground motion. Hu Heng [7], based on the existing research and combined with Zhu Meizhen's prediction method of highway bridge seismic damage, established an improved prediction model of highway subgrade and pavement seismic damage. The practicality of it is verified using Wenchuan earthquake data. It should be pointed out that the improved method of earthquake damage prediction has obviously improved the accuracy of the results, but in order to simplify the calculation procedure, an exact bound division is used to classify the damage grades.

Considering the uncertainty of earthquake occurrence, the randomness of earthquake damage phenomena and the fuzziness of disaster degree and other factors [1], a membership function of fuzzy subset of failure grade is established in this paper, then using closeness degree method, the fuzzy recognition for membership function of earthquake damage is conducted. The results of earthquake damage prediction are more realistic. Finally, an example is given by using the earthquake damage data of Wenchuan earthquake. The results show that the accuracy of the calculation is indeed improved.

\section{Earthquake Damage Estimation of Highway Subgrade and Pavement}

\subsection{Improved Methodology for Seismic Estimation of Highway}

In order to simplify the work, Chen Yiping's method used three levels of seismic damage classification. The improved method uses a more reasonable five-level division, this is consistent with other lifeline systems damage grades of five levels. The earthquake damage grades of highway structures are divided into five grades: basically intact, slightly damaged, moderately damaged, severely damaged and damaged in Classification of earthquake damage to lifeline engineering (GB/T24336-2009). And referring to the China Earthquake Intensity Table (GB/T 17742-2008) on the different damage levels under the earthquake damage index division.

It can be seen that the value of dividing the grade limit is very clear. Hence, situation like the calculated result is only different by 0.01 , but the damage level will change from slight damage to medium damage will be inevitable, which is not realistic. In fact, the above description of the classification of earthquake damage is a typical fuzzy concept [8]. Therefore, it is more reasonable to conform to fuzzy judgment.

Table 1. Highway engineering earthquake damage index and its feature.

\begin{tabular}{|c|c|c|}
\hline Damage Degree & Feature & Damage Index \\
\hline Basically Intact & Pavement and embankment with no or little damage. Passable & $-0.00,0.10)$ \\
\hline Slight Damage & $\begin{array}{l}\text { Shoulder, retaining wall, road surface and cutting with fine cracks. There may be a slight subsidence or uplift } \\
\text { (less than } 15 \mathrm{~cm} \text { ). Passable with some traffic obstacles. }\end{array}$ & {$[0.10,0.30)$} \\
\hline Medium Damage & $\begin{array}{l}\text { There appears small inhomogeneous collapse on roadbed. Passing is possible but falling slopes and rolling stones } \\
\text { should be noticed. And repairing is still needed. }\end{array}$ & {$[0.30,0.55)$} \\
\hline Serious Damage & $\begin{array}{l}\text { Obvious and inhomogeneous collapse appear on roadbed. It's hard to pass, besides, traffic limitation and } \\
\text { overhaul are definitely needed. }\end{array}$ & {$[0.55,0.85)$} \\
\hline
\end{tabular}

In this paper, the seismic damage index $\overline{i n d}_{i}$ of section number $i$ of pavement is calculated by using the improved prediction model of subgrade and pavement.

The calculation formula is as follows:

$$
\overline{\operatorname{ind}}_{i}=c_{0} \prod_{j=1}^{n} \prod_{k=1}^{r_{j}} c_{j k}{ }_{i j k}^{x_{i j k}}
$$

The regression values and recommended values of the normalized factor coefficients can be obtained by consulting the tables. The regression value and recommended value of $c_{0}$ are 0.013785 and 0.0138 respectively.

The degree of earthquake damage of highway subgrade and pavement can be expressed by seismic damage degree.

Earthquake damage degree is a fuzzy set based on earthquake damage index. Based on the experience of earthquake damage and from the convenience of calculation and analysis, the earthquake damage degree is a normal distribution curve [1]:

$$
\mu_{i}\left(\text { ind }_{i}\right)=\frac{1}{\sqrt{2 \pi} \sigma_{i}} e^{\frac{-\left(\text { ind }_{i}-\overline{\text { ind }}\right)}{2 \sigma_{i}^{2}}}
$$

In conclusion, the membership function curve of seismic damage degree of the section number of pavement can be calculated, that is, the fuzzy set to be identified

\subsection{Determination of Membership Function of Damage Grades}

Seismic damage occurrence of highway structures is a gradual process, from minor local damage to more serious overall damage. Therefore, the fuzzy subset $B_{1} \sim B_{5}$ corresponding to the destruction grade is divided according to the grade limit value given in the table above. Referring to the real earthquake damage data, the membership function of each subset is determined by reference 9, which are as 
follow:

$$
\begin{gathered}
\mu_{B_{1}}\left(\text { ind }_{i}\right)=\frac{1}{2}\left[\sin \left(10 \text { ind }_{i}\right) \pi+1\right] \\
\text { ind }_{i} \in[0.00,0.10) \\
\mu_{B_{2}}\left(\text { ind }_{i}\right)=\frac{1}{2}\left[\sin \left(\text { ind }_{i}-0.5\right) \pi+1\right] \\
\text { ind }_{i} \in[0.10,0.30) \\
\mu_{B_{3}}\left(\text { ind }_{i}\right)=\frac{1}{2}\left[\sin \left(4 \text { ind }_{i}-1.2\right) \pi+1\right] \\
\text { ind }_{B_{4}} \in[0.30,0.55) \\
\text { ind } \left._{i}\right)=\frac{1}{2}\left[\sin \left(\frac{10}{3} \text { ind }_{i}-\frac{10}{3}\right) \pi+1\right] \text { ind }_{i} \in[0.55,0.85) \\
=\frac{1}{2}\left[\sin \left(\frac{20}{3} \text { ind }_{i}-\frac{17}{3}\right) \pi+1\right] \text { ind }_{i} \in[0.85,1.00)
\end{gathered}
$$

Here is the standard fuzzy set.

\subsection{Determining Earthquake Damage Grade by Fuzzy Recognition Using Closeness Degree Method}

An exact bound division is used to classify the damage grades in the improved methodology for seismic estimation of highway. The $\overline{i n d}_{i}$ value of a certain road surface is then calculated, and the result of seismic damage estimation damage can be obtained by comparing the grading table. This method is intuitive, simple and practical. However, to some extent, it is necessary to make the boundaries clear and fuzzy. In order to further improve the accuracy of the results, this paper decided to adopt the method of closeness degree.

The concept of degree of closeness is given as: If A and B are fuzzy subsets in the universe $U$, the degree of closeness of two fuzzy sets can be described by the closeness degree.

The closeness degree being often used includes symmetry closeness degree、 asymmetric closeness degree、 euclidean approach degree, lattice closeness degree, etc. In order to simplify the calculation process, we choose to use the lattice closeness degree to judge the closeness of two certain fuzzy sets to improve the earthquake damage estimation method. According to its definition, the greater the lattice closeness degree, the closer the two fuzzy sets are.

The formula for calculating the degree of lattice closeness is as follows:

$$
\sigma_{0}(\underset{\sim}{A}, B)=\frac{1}{2}[\underset{\sim}{A} \underset{\sim}{ } B+(1-\underset{\sim}{A} \underset{\sim}{\odot})]
$$

Among them, $[\underset{\sim}{A \circ B} \sim \underset{\sim}{\sim}]$ is called inner product, and $[\underset{\sim}{A \odot B} \underset{\sim}{\sim}]$ is called outer product. In fact, there are many methods to calculate the degree of closeness between fuzzy sets. Here, the lattice degree of closeness is more convenient to calculate.

For a certain section of pavement, the value of each influence factor can be obtained according to the actual structure information to calculate the $\overline{\text { ind }}_{i}$ value, and then the subordinate function of seismic damage degree can be obtained. Then calculate the lattice closeness degree of membership function with the subset of the above damage levels. Now presenting the example.

\section{Example}

Referring to the earthquake damage data of Wenchuan earthquake, we calculated a certain section of the damaged National Highway 213. The section is a third-class highway, belongs to class II site, which is with semi-excavation and semi-filling subgrade, grouting gravity retaining, slope height less than 15 meters, general protection and seismic performance, subgrade height difference 6 meters, the fortification intensity is 7-degree. Based on the above information, the table shows the values of each damage factor as shown in Table 2.

Table 2. Earthquake estimation parameters'value.

\begin{tabular}{lllll}
\hline $\boldsymbol{i}$ & Parameters & Type & $\boldsymbol{j}$ & Value \\
\hline 1 & Highway grade & Grade-III & 1 & 1.14 \\
2 & Subgrade type & Part-cut and part-fill & 2 & 2.00 \\
3 & Retaining wall type & Concrete gravity & 3 & 1.62 \\
4 & soil type & II & 4 & 1.05 \\
5 & slope height & 9.7 & 5 & 1.07 \\
6 & slope gradient & $35^{\circ} \leq \theta \leq 65^{\circ}$ & 6 & 1.19 \\
7 & side slope protection & Seismic favorable & 7 & 1.06 \\
8 & Subgrade elevation difference & 6 & 8 & 1.23 \\
9 & Seismic fortification intensity & 7 & 9 & 1.67 \\
10 & actual intensity & 11 & 10 & 3.43 \\
\hline
\end{tabular}


The above data are taken into the calculation formula to calculate the $\overline{i n d}_{i}$ value of the section:

$$
\overline{\operatorname{ind}}_{i}=c_{0} \prod_{j=1}^{n} \prod_{k=1}^{r_{j}} c_{j k}{ }^{x_{i j k}}=0.5089
$$

The average damage index of the section is 0.5089 , and the membership function of seismic damage is as follows:

$$
\mu_{i}\left(\text { ind }_{i}\right)=\frac{1}{\sqrt{2 \pi} \sigma_{i}} e^{\frac{-\left(\text { ind }_{i}-0.5089\right)}{2 \sigma_{i}^{2}}}
$$

Next, the closeness degree of membership functions of each fuzzy set is calculated. Taking the membership function of $\mu_{B_{3}}\left(\right.$ ind $\left._{i}\right)$ as an example, the following membership functions are expressed as follows:

$$
\mu_{B_{3}}\left(\text { ind }_{i}\right)=\frac{1}{2}\left[\sin \left(4 \text { ind }_{i}-1.2\right) \pi+1\right] \text { ind }_{i} \in[0.30,0.55)
$$

Calculating the inner product:

$$
\left[\underset{\sim}{\mu_{i}\left(\text { ind }_{i}\right) \circ \mu_{B_{3}}\left(\text { ind }_{i}\right)}\right]=0.9729
$$

This part makes a brief summary of the whole paper. Usually, there should be one paragraph in this part.

External product:

$$
\left[\underset{\sim}{\left.\mu_{i}\left(\text { ind }_{i}\right) \odot \underset{B_{3}}{\mu_{\sim}} \text { ind }_{i}\right)}\right]=0
$$

Finally, we get the lattice closeness degree value of the two:

$$
\underset{\sim}{\sigma_{0}\left(\mu_{i}\left(\text { ind }_{i}\right), \mu_{B_{3}}\left(\text { ind }_{i}\right)\right)}=\frac{1}{2}\left[\left[\underset{\sim}{\mu_{i}\left(\text { ind }_{i}\right) \circ \mu_{B_{3}}\left(\text { ind }_{i}\right)}\right]+\left(1-\left[\underset{\sim}{\mu_{i}\left(\text { ind }_{i}\right) \odot \mu_{B_{3}}\left(\text { ind }_{i}\right)}\right]\right)\right]=0.9864
$$

Closeness degree values between the membership function of the same computable earthquake damage degree and the other four fuzzy sets of the earthquake damage grade are calculated:

$$
\begin{aligned}
& \sigma_{0}\left(\mu_{i}\left(\text { ind }_{i}\right), \mu_{B_{1}}\left(\text { ind }_{i}\right)\right)=0 \\
& \sigma_{0}\left(\mu_{i}\left(\text { ind }_{i}\right), \mu_{B_{2}}\left(\text { ind }_{i}\right)\right)=0.8271 \\
& \sigma_{0}\left(\mu_{i}\left(\text { ind }_{i}\right), \mu_{B_{4}}\left(\text { ind }_{i}\right)\right)=0.9979 \\
& \sigma_{0}\left(\mu_{i}\left(\text { ind }_{i}\right), \mu_{B_{5}}\left(\text { ind }_{i}\right)\right)=0.9689
\end{aligned}
$$

Here:

$$
0.9979>0.9864>0.9689>0.8271>0
$$

Therefore, we can see that the grade of earthquake prediction result of this certain section using the method of closeness degree is seriously damaged.

And:

\section{$0.30<0.5089<0.55$}

So we can see that the result of using the improved earthquake damage prediction method is medium damage.

Obviously, the final earthquake damage grade is serious damaged, which is consistent with the actual earthquake damage result. However, the result obtained by using the improved earthquake damage estimating method is of medium damage, which is one order different from the actual damage level of the section. It can be seen that the accuracy of the improved method of subgrade and pavement earthquake damage estimating using the closeness degree method is higher than that of the original method, which also proves its effectiveness.

\section{Conclusion}

China is a country with high seismic risk. We all understand the suddenness and serious consequences of earthquake disasters. If the damage degree can be predicted more accurately in advance, the rescue plan can be formulated more quickly and rationally after the disaster, so as to reduce the loss of life and property of the people to the greatest extent. Therefore, a more accurate method of earthquake damage prediction is necessary. Through the example verification, the improved method of earthquake damage prediction in this paper has higher accuracy. In this paper, the lattice closeness degree is used, which simplifies the calculation, henceforth, in future research, the choice of progress schedule should be further considered.

\section{Acknowledgements}

This work was financially supported by the special fund for basic scientific research business of central public research institutes (2018A02) from Institute of Engineering Mechanics, China Earthquake Administration.

\section{References}

[1] Yi-ping Chen, Xin Chen, Seismic damage prediction of subgrade and pavement, J, Earthquake Resistant Engineering. 3 (1993) 010.

[2] Xu Wei, Highway damage in California earthquake, United States, J, China Civil Engineering Journal. 2 (1992) 012. 
[3] Jinsong Jiang, '5.12' Wenchuan earthquake Sichuan highway damage and Enlightenment, J, Hubei road traffic science and technology. 3 (2008) 024.

[4] Stefan Unterrader and Sven Fuchs. Quantifying rockfall risk on roads in the Port Hills, Christchurch, New Zealand. Geophysical Research Abstracts Vol. 18, EGU2016-5, 2016.

[5] LIU Jinlong et al., Investigation and analysis of seismic damage of highway system in Lushan " 4.20 " earthquake, J, Journal of Natural Disasters. 3 (2013) 022.

[6] JIA Xingli, XU Jinliang, Cloud Model-based Seismic Risk Assessment of Road in Earthquake Region, J, Journal of Tongji University (Natural Science). 9 (2014) 042.

[7] Heng Hu, Junqi Lin, and Jinlong Liu. Improved Methodology for Seismic Estimation of Highway Based on Wenchuan Earthquake. 2018 International Conference on Modeling, Simulation and Optimization. 2018.
[8] Chen Tuyun, Liu Xihui, Study on the concept of earthquake damage grade by fuzzy partition, J, South China Journal of Seismology. 4 (1984).

[9] Xie Jijian, Liu Chengping, Fuzzy mathematics method and its application, Huazhong University of Science and Technology Press. Wuhan, China, 2015.

[10] Yoshihisa Maruyama, Fumio Yamazaki, Fragility curves for expressway embankments based on damage datasets after recent earthquakes in Japan, J. Soil Dynamics and Earthquake Engineering, 30(2010) 1158-1167.

[11] Classification of earthquake damage to lifeline engineering (GB/T24336-2009).

[12] China Earthquake Intensity Table (GB/T 17742-2008). 